\title{
Pediatric Gastrointestinal Motility - Future Directions and Challenges
}

\author{
Nader N. Youssef ${ }^{a}$ Carlo Di Lorenzo ${ }^{b}$ \\ a Division of Pediatric Gastroenterology, Goryeb Children's Hospital/Atlantic Health System/University of \\ Medicine and Dentistry of New Jersey Morristown, Morristown, N.J., and ${ }^{b}$ Division of Pediatric Gastroenterology, \\ Columbus Children's Hospital/The Ohio State University Columbus, Columbus, Ohio, USA
}

\section{Key Words}

Pediatric gastrointestinal motility •

Neurogastroenterology $\cdot$ Pharmacogenomics $\cdot$ Children

\begin{abstract}
The study of gastrointestinal motility has evolved to a sophisticated diagnostic technique that is widely used clinically to further guide management of children with complex gastrointestinal problems. Thorough comprehension requires a multidisciplinary approach with the integration of molecular and cell biology, organ physiology, and clinical observations. During the past decade there has been a dramatic increase in our knowledge of the enteric neuromuscular system. Continued exploration of targeted gene mutations in animal models has the potential of enhancing our understanding of congenital disorders of gastrointestinal motility. Experiments studying polymorphisms in serotonin transporter gene (SERT) and different therapeutic responses to serotonergic agents in adults with irritable bowel syndrome need to be carried out in children with functional bowel disorders. Additional considerations that need to be addressed if advances are to continue include increasing the number of specialists interest in motility disorders and identifying funding sources to support the establishment of research consortiums among pediatric centers.
\end{abstract}

\section{KARGER}

Fax +4161306 1234 E-Mail karger@karger.ch www.karger.com

\section{Introduction}

The study of gastrointestinal motility has evolved from an esoteric laboratory tool to a sophisticated diagnostic technique that is now widely used clinically to guide management of children with complex gastrointestinal problems [1]. Previously, many gastrointestinal motility disorders were ignored or attributed to emotional disturbances. With the growing understanding of how gastrointestinal muscle and nerve dysfunctions are directly linked to symptom generation in individuals of all ages, great strides have been made in the approach to the diagnosis and treatment of pediatric gastrointestinal motility disorders.

A thorough comprehension of pediatric gastrointestinal motility requires a multidisciplinary approach with the integration of molecular and cell biology, organ physiology, and clinical observations. Smooth muscle cells, enteric neurons, interstitial cells of Cajal, hormones, gutbrain pathways, and the central nervous system continuously interact with each other. Social, cultural, emotional, and developmental factors influence symptom generation and healthcare use. All these factors are currently being actively investigated by a cadre of pediatric investigators [2-4]. This interaction of multiple disciplines is defining a new specialty - neurogastroenterology - and observations made within the pediatric age group, including the role of early life events, the importance of genetics 
and the environment, and natural history of childhood functional bowel disorders are beginning to have an impact in the way adult gastroenterologists view and treat gut motor and sensory disturbances $[5,6]$.

\section{Future Directions}

During the past decade there has been a dramatic increase in our understanding of the complex structure, function, and developmental biology of the enteric neuromuscular system $[7,8]$. Nuclear and mitochondrial mutations associated with abnormalities of neuroenteric development have been identified in animal models and human diseases. These mutations result in a spectrum of disorders: aganglionosis, absence or heterotopia of specific neuronal cell populations, lack of interstitial cells of Cajal, and dysfunctional intestinal smooth muscle. A classification of motility disorders acknowledging congenital or developmental abnormalities needs to be developed.

Continued exploration of the functional and morphological consequences of targeted gene mutations in animal models has the potential of enhancing our understanding of the causes of congenital and acquired disorders of gastrointestinal motility [9]. To date, only a few laboratories have the expertise or financial support to perform the sophisticated studies required to identify specific gene mutations. Establishment of uniform methods to characterize specific defects in tissue samples from patients with well-characterized gastrointestinal motility disorders will allow linking those defects to phenotypic expressions. This will enhance our ability to classify patients so that accurate prognostic information and more targeted interventions can be developed.

Much needs to be learned from a careful classification of different histopathologic findings. One of the most significant shortcomings in the evaluation of the enteric nervous system has been the lack of uniform methodology to study pathologic specimens obtained from patients with enteric neuromuscular disorders. Although full-thickness biopsies are often obtained, most pathology protocols routinely utilize diagnostic techniques focused on hematoxylin and eosin staining on paraffin sections and not specifically on the evaluation of the enteric nervous system. In addition, pediatric site- and age-specific tissue controls are often lacking. Evaluation of a specimen obtained from the proximal bowel of a few months old patient cannot be compared to a specimen obtained from the distal bowel of an adolescent control without risking misinterpretations. Few central laboratories ('tissue banks') with a keen interest in disorders of gastrointestinal motility should be established to systematically study the anatomic structure
Table 1. Some of the current challenges in pediatric gastrointestinal motility $[11,14]$

1 Define mechanisms leading to feeding intolerance in preterm babies

2 Evaluate oromotor dysfunction and feeding aversion in the older child and in neurologically impaired children

3 Characterize the pathogenesis of post-fundoplication complications

4 Characterize the pathophysiology of chronic pseudoobstruction and evaluate role of diagnostic techniques and benefit of potential treatments

5 Characterize the pathophysiology diagnostic techniques and potential treatments of defecation disorders, including functional constipation, Hirschsprung's disease, and other colonic neuromuscular disorders

6 Evaluate pathophysiological mechanisms and potential interventions in patients with cyclic vomiting syndrome

7 Identify histopathologic and ultrastructural features of neurocristopathies and other linkage defects of intestinal nerves and muscles

8 Identify genetic markers of neurocristopathies

9 Investigation of enteric neurobiology and development of the enteric nervous system

10 Investigation of the neuroimmunology of the gut: characterization of the interaction between the central nervous system, enteric nervous system, and immune system

11 Evaluation of the role of early life events in the development of motility and functional gastrointestinal disorders

12 Creation of a tissue bank for children with enteric neuromuscular disorders

of the enteric nervous system, interstitial cells of Cajal, and gastrointestinal smooth muscles in samples obtained from children with well-defined motor disorders. Such laboratories should collaborate to develop uniform protocols for investigation of submitted tissue samples.

Pharmacogenomics, the study of how an individual's genetic inheritance affects the body's response to drugs needs to be developed in pediatrics. Currently, treatment of pediatric inflammatory bowel diseases is benefiting from the evaluation of the enzyme thiopurine methyltransferase, allowing for safer and more effective use of thiopurines. Preliminary studies in adults with irritable bowel syndrome have suggested that polymorphisms in serotonin transporter gene (SERT) are associated with different therapeutic responses to serotonergic agents [10]. Similar studies need to be performed in children with functional bowel disorders, a population of patients likely to have fewer confounding factors (associated physical and psychological disorders) than their adult counterpart. Table 1 lists other areas for future studies having highest priority in the field of motility and functional 
bowel disorders as identified by the Working Group Report of the 2nd World Congress of Pediatric Gastroenterology, Hepatology, and Nutrition [11].

\section{How Do We Accomplish Our Goals?}

Funding sources must be identified to support the establishment of novel methodologies to screen genetically altered animal as models for gastrointestinal motility disorders. Potential funding sources might include both federal agencies and pharmaceutical companies. The National Institute of Diabetes and Kidney Disease (NIDDK), through its Division of Digestive Diseases and Nutrition, and the American Digestive Health Foundation (ADHF), recently issued a request for application (RFA) [12]. The intent of this RFA was to (1) encourage interaction between basic scientists and clinical investigators with special interest in motility-related disorders and (2) encourage collaborative interactions among gastroenterologists and neuroscientists. The overall scientific goals of the initiative were to enhance the understanding of the genetic molecular, cellular, and physiological changes that occur in the bowel wall, central brain nuclei, and peripheral autonomic ganglia innervating the gastrointestinal tract in persons and animal models with motility-related disorders. Secondary goals included definition of the biochemical coding of stress-related alterations in gastrointestinal motility, physiological and cognitive influences on course of motility disorders, food-related motor disturbances, and the ontogeny at the genetic, molecular, and cellular levels of lifelong motility disorders. The RFA was a tremendous success with a large amount of excellent quality research and stimulated further research in the field and represented a promising initial step. It is auspicable that in the future similar RFA be targeted specifically for studies in pediatrics.

\section{What Are the Obstacles to Be Overcome?}

Currently, in the USA there is a shortage of pediatric gastroenterologists with a specific interest in motility disorders. It is encouraging though, that at recent national and international meetings there has been an increased amount of excellent clinical and basic research presented by clinicians with an interest in functional bowel disorders in children. A handful of centers are now qualified to train physicians to reach 'level 2' competence in gastrointestinal motility as per the North American Society Pediatric Gastroenterology Hepatology, and Nutrition (NASPGHAN) Training Guidelines [13]. Improved training and understanding of enteric pathophysiology may result in significant financial savings if one considers the huge financial burden of parenteral nutrition and small bowel transplants, which are utilized by children with the most severe forms of motility disorders. It has been estimated that if every preterm infant in the USA can have 1 week less of parenteral nutrition secondary to feeding intolerance, the estimated annual savings in healthcare expenditures would approximate 1 billion dollars [14].

Development of diagnostic tests more suitable for use in young individuals needs to be considered a high priority. Techniques involving catheters, balloons or radiations are difficult to perform in newborns and infants and are unsuitable in healthy children, making it impossible to have normal values to be used as controls. Less invasive tests to assess gastric sensation and accommodation, such as the nutrient or water load test [15], breath tests using ${ }^{13} \mathrm{C}$-Spirulina platensis breath test for gastric emptying of solids [16] and single photon emission computed tomography (SPECT) [17], need to be further developed and validated in children.

Another problem that pediatric gastroenterologists face on a daily basis is the lack of medications with proven efficacy in children with motility disorders. In the USA, children are commonly treated with drugs that have not been approved for pediatric use by the FDA. In 1962, the Kefauver-Harris amendments to the Food, Drug, and Cosmetic Act were designed to ensure safety and effectiveness for human drug use. However, infants and children were excluded from the protections that these amendments were to provide. Once a drug was approved for use by the FDA, further studies to determine safety and efficacy in infants and children were rarely conducted [18]. Seventy-five percent of all drugs prescribed for children have never been tested on children. Doses are extrapolated from adult studies resulting often in inappropriate dosing in pediatrics [19], a problem that has inspired major policy changes in recent years. In 1997, the FDA established its Pediatric Rule. Under the rule, if the agency believes that a new drug will have significant pediatric use, it can order the manufacturer to carry out pediatric clinical trials before the drug can be marketed. Also, in 1997, Congress passed the FDA Modernization Act (FDAMA). The act offers drug companies a 6-month patent extension as an incentive to conduct pediatric trials. For regulatory purposes, pediatric is defined as between the ages of 2 and 18. The issue of the lack of pediatric drug data is important because effective treatment may be withheld or children may be treated with medications without a full understanding of the risks, benefits, and implications. 
Progress in the field of therapy of pediatric motility disorders has also been slow in the past due to the fact that there have been very few well designed and controlled treatment studies in children. As a result, the pediatric gastrointestinal literature abounds in case series, retrospective reviews and small pathophysiologic or epidemiologic studies difficult to interpret and of limited significance. Efforts are currently underway to create infrastructures aimed at facilitating multi-center, collaborative studies, involving clinicians, basic scientists, academic and non-academic physicians and healthcare professionals from developed and developing countries. In this way, trials would be adequately powered leading to more meaningful and more generalizable data. Establishment of clinical research networks may increase our ability to attract the elusive research funds targeted for the study pediatric gastrointestinal motility disorders.

Definition of entry criteria to enroll patients in pediatric clinical trials has also been a problem in the past. The pediatric Rome Criteria for functional gastrointestinal disorders were only published in 1999 [20]. These criteria were meant to be guidelines to help identify children with functional disorders in an attempt to define subsets of children with recurrent clusters of symptoms. To date there have been conflicting data on the utility of these criteria. There is evidence of both poor inter- and intra-rater reliability when trying to apply these criteria [21]. Investigators who have tried to validate the pediatric Rome Criteria with age appropriate gastrointestinal questionnaires found that only $55 \%$ of children met one of the criteria [22]. Other obstacles have included the use of the word 'functional' to describe the nature of many of these disorders. There continues to be a great deal of confusion and misunderstanding, among patients and physicians who deal with functional bowel disorder. This lack of understanding can lead to misdiagnosis and misguided treatment. It has been reported that those patients that have been labeled with a functional gastrointestinal disorder have more surgeries than similar control populations [23].

Gaining public support for pediatric drug research can be difficult as patients often feel 'used' for academic research when communication is not clear between subjects and researchers [24]. One survey showed parents did not realize the drug studies involving their children were to assess not only for efficacy, but for safety as well. Only one-third of the parents knew they could withdraw their child from the study at anytime. Others responded that the informed consent was unnecessary because they would do whatever the doctor recommended [25]. In order to obtain the support of the general population for pediatric drug testing, consumers must be better informed of the process. Most institutional review boards for pediatric research require assent and consent forms to be signed by the child and the parents respectively. The question of determining the age when a child is old enough to consent to participate or to choose to dropout or continue participation in drug studies is not solved yet. Researchers often conclude that there are simply not enough potential patients to participate in pediatric research. Multi-site studies should be encouraged to ensure sufficient pediatric participation.

Other challenges in the study of pediatric gastrointestinal motility disorders include the difficulty of performing prospective longitudinal studies in order to determine long-term outcomes of pediatric patients due the high mobility of the US population. Such studies so far have been accomplished in Europe where language barriers and cultural factors keep generations of families in the same geographic areas [26], and may not necessarily be applicable to US patients. New rules such as the Health Insurance Portability and Accountability Act of 1996 (HIPAA) intended to protect health insurance coverage for workers and their families when they change or lose their jobs have led to significant privacy concerns both by hospital administrators and patients and have increased the complexity of performing clinical studies in children.

\section{Conclusion}

The field of pediatric gastrointestinal motility has a brighter future, due to our improved ability to study biologic systems from isolated cells to organ function. Studies of the role and development of interstitial cells of Cajal are under way and may clarify the origin of motor rhythmicity. The interaction between gastrointestinal neuromuscular activity and perception of symptoms is another area that is currently actively pursued by clinical and laboratory investigators. Evaluation of the association between gastrointestinal neuromuscular dysfunction and symptoms has the potential to lead to the development of new diagnostic tests and pharmacological and nonpharmacological interventions. Funding dictates where research efforts will be directed and pediatric studies because of their inherent ethical and practical difficulties have a history of being underfunded despite the fact that 
many motility disorders diagnosed in adults have their roots in events occurring during childhood or were already present early on in life. With adequate financial support given to investigators with an interest in pediatric gut disorders, significant enhancements will be made in our understanding of the enteric nervous system function and the evaluation and treatment of motility disorders from childhood to adulthood.

\section{References}

1 Pensabene L, Youssef NN, Griffiths J, Di Lorenzo C: Colonic manometry in children with defecatory disorders. Role in diagnosis and management. Am J Gastroenterol 2003;5: 1052-1057.

- 2 Hutson JM, Chow CW, Hurley MR, Wheatley JM, Catto-Smith AG: Deficiency of substance P-immunoreactive nerve fibres in children with intractable constipation: a form of intestinal neuronal dysplasia. J Paediatr Child Health 1997;3:187-189.

- 3 Meier-Ruge WA, Holschneider AM, Scharli AF: New pathogenetic aspects of gut dysmotility in aplastic and hypoplastic desmosis of early childhood. Pediatr Surg Int 2001;2:140143.

-4 Sabri MT, Barksdale E, Di Lorenzo C: Constipation and lack of colonic interstitial cells of Cajal. Dig Dis Sci 2003;5:849-853.

5 Levy RL, Jones KR, Whitehead WE, Feld SI, Talley NJ, et al: Irritable bowel syndrome in twins: heredity and social learning both contribute to etiology. Gastroenterology 2001; 4 : 799-804.

6 Coutinho SV, Plotsky PM, Sablad M, Miller JC, Zhou H, et al: Neonatal maternal separation alters stress-induced responses to viscerosomatic nociceptive stimuli in rat. Am $\mathrm{J}$ Physiol 2002;2:G307-G316.

-7 Gershon MD: Serotonin receptors and transporters - roles in normal and abnormal gastrointestinal motility. Aliment Pharmacol Ther 2004;S7:3-14.

8 Talley NJ: Review article: 5-hydroxytryptamine agonists and antagonists in the modulation of gastrointestinal motility and sensation: clinical implications. Aliment Pharmacol Ther 1992;3:273-289.
9 Gestblom C, Sweetser DA, Doggett B, Kapur RP: Sympathoadrenal hyperplasia causes renal malformations in Ret (MEN2B)-transgenic mice. Am J Pathol 1999;6:2167-2179.

10 Park MI, Camilleri M: Genetics and genotypes in irritable bowel syndrome: implications for diagnosis and treatment. Gastroenterol Clin North Am 2005;34:305-317.

11 Di Lorenzo C, Benninga MA, Forbes D, Morais $\mathrm{CB}$, Morera $\mathrm{C}$, Rudolph $\mathrm{C}$, et al: Functional gastrointestinal disorders, gastroesophageal reflux and neurogastroenterology: Working Group report of the second World Congress of Pediatric Gastroenterology, Hepatology, and Nutrition. J Pediatr Gastroenterol Nutr 2004; 39:S616-S625.

$12 \mathrm{http} / /$ grants.nih.gov/grants/guide/rfa-files/ RFA-DK-99-004.html

-13 Rudolph CD, Winter HS: NASPGN guidelines for training in pediatric gastroenterology. NASPGN Executive Council, NASPGN Training and Education Committee. J Pediatr Gastroenterol Nutr 1999;29:S1-S26.

14 Li BU, Altschuler SM, Berseth CD, Di Lorenzo C, Rudolph CD, et al: Research Agenda for Motility and Functional Disorders: Report of NASPGHAN to the Children's Digestive Heath Foundation. J Pediatr Gastroenterol Nutr 2003;35:S263-S267.

15 Sood MR, Schwankovsky LM, Rowhani A, Zangen T, Ziring D, Furtado T, et al: Water load test in children. J Pediatr Gastroenterol Nutr 2002;35:199-201.

16 Chitkara DK, Camilleri M, Zinsmeister AR, Burton D, El-Youssef M, Freese D, et al: Gastric sensory and motor dysfunction in adolescents with functional dyspepsia. J Pediatr 2005; 146:500-505.
17 De Schepper DU, Cremonini F, Chitkara DK, Camilleri M: Assessment of gastric accommodation: overview and evaluation of current methods. Neurogastroenterol Motil 2004;16: 275-285.

18 Kauffman RE: Status of drug approval processes and regulation of medications for children. Curr Opin Pediatr 1995; 7:195-198.

19 Bonetta L: Pediatric study of medicines becomes mandatory. Nat Med 2000;10:1069.

20 Rasquin-Weber A, Hyman PE, Cucchiara S, Fleisher DR, Hyams JS, Milla PJ, et al: Childhood functional gastrointestinal disorders. Gut 1999;45(suppl 2):60-68.

21 Saps M, Di Lorenzo C: Interobserver and intraobserver reliability of the Rome II criteria in children. Am J Gastroenterol 2005;100: 2079-2082.

22 Caplan A, Walker L, Rasquin A: Validation of the Pediatric Rome II Criteria for Functional Gastrointestinal Disorders Using the Questionnaire on Pediatric Gastrointestinal Symptoms. J Pediatr Gastroenterol Nutr 2005;3: 305-316.

23 Burns DG. The risk of abdominal surgery in irritable bowel syndrome. S Afr Med J 1986;2: 91.

24 Vitiello B, Jensen PS: Medication development and testing in children and adolescents. Arch Gen Psychiatry 1997;54:871-876.

25 Harth SC, Thong, YH: Parental perceptions and attitudes about informed consent in clinical research involving children. Soc Sci Med 1995;41:1645-1651.

26 Van Ginkel R, Reitsma J, Buller H, van Wijk MK, Taminiau JA, Benninga MA: Childhood constipation: longitudinal follow-up beyond puberty. Gastroenterology 2003;125:357363. 